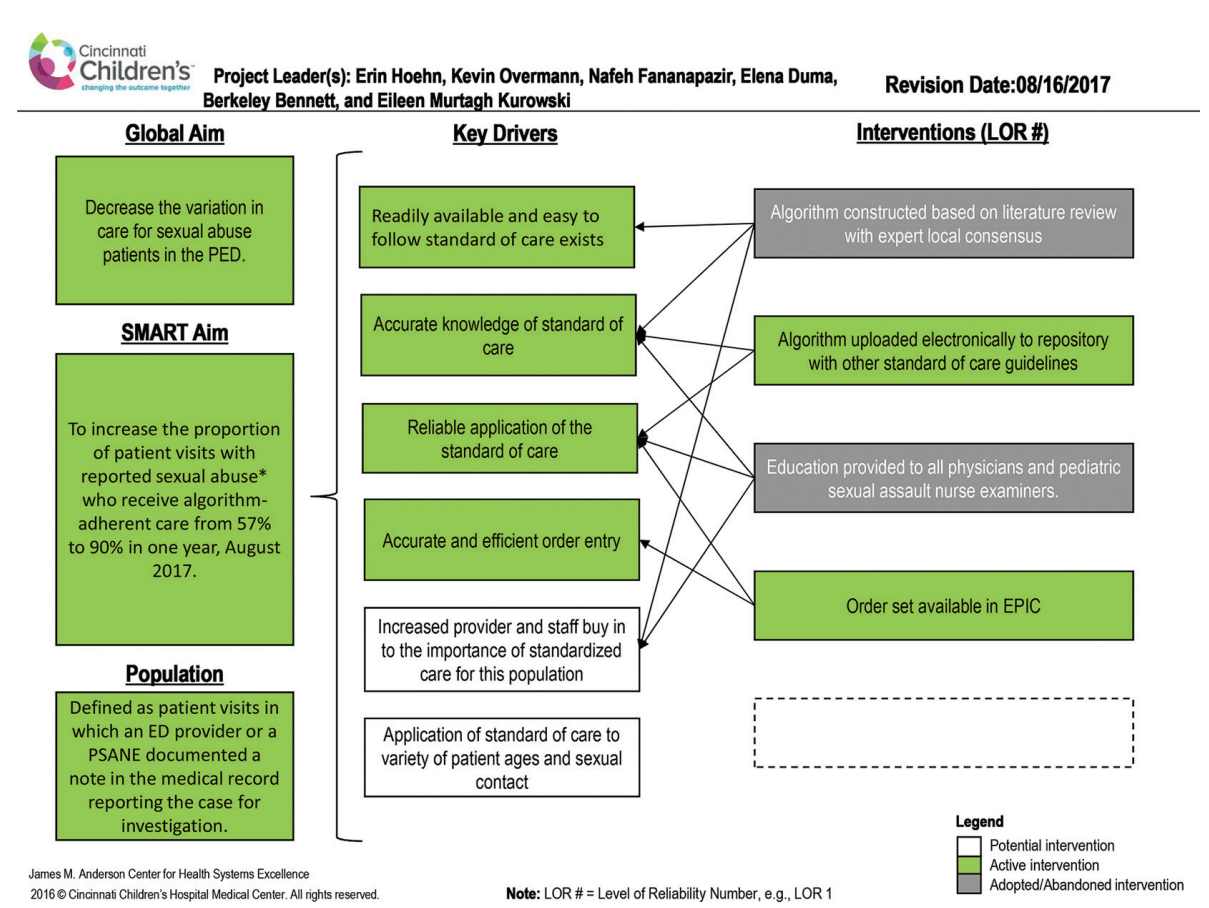

Abstract 985 Figure 1 Evaluation of sexual abuse key driver diagram (KDD)

research on evaluation of health initiatives (Craig et al., 2008; Parry et al., 2013), we recommend the use of formative evaluation as means to support learning while implementation is taking place as a means to increase the likelihood of reaching outcome and making progress toward health, wellness, and equity.

\section{QUALITY IMPROVEMENT INTERVENTIONS INCREASE ADHERENCE TO GUIDELINE FOR EVALUATION AND TREATMENT OF SEXUAL ABUSE VICTIMS IN A PAEDIATRIC EMERGENCY DEPARTMENT}

Erin Hoehn, Kevin Overmann, Nafeh Fananapazir, Berkeley Bennett, Elena Duma, Eileen Murtagh Kurowski. Cincinnati Children's Hospital Medical Centre, US

10.1136/bmjoq-2017-IHI.19
Background Appropriate medical care of sexual abuse victims who present to the paediatric emergency department (PED) is vital to facilitate forensic evidence collection and prevent pregnancy and sexually transmitted infections. Despite recommendations from the American Academy of Paediatrics and Centres for Disease Control, adherence to guidelines remains low.

Objectives We aimed to increase the proportion of patient encounters at a PED for reported sexual abuse that receive guideline-adherent care from $57 \%$ to $90 \%$ within 12 months. Methods Our team of PED and child abuse paediatricians constructed a key driver diagram to outline our theory for improvement (Figure 1). Multiple plan-do-study-act cycles were conducted to test interventions aimed at key drivers, including construction of a best practice algorithm, targeted clinician education, and integration of an electronic order set. Our primary outcome was the proportion of patient

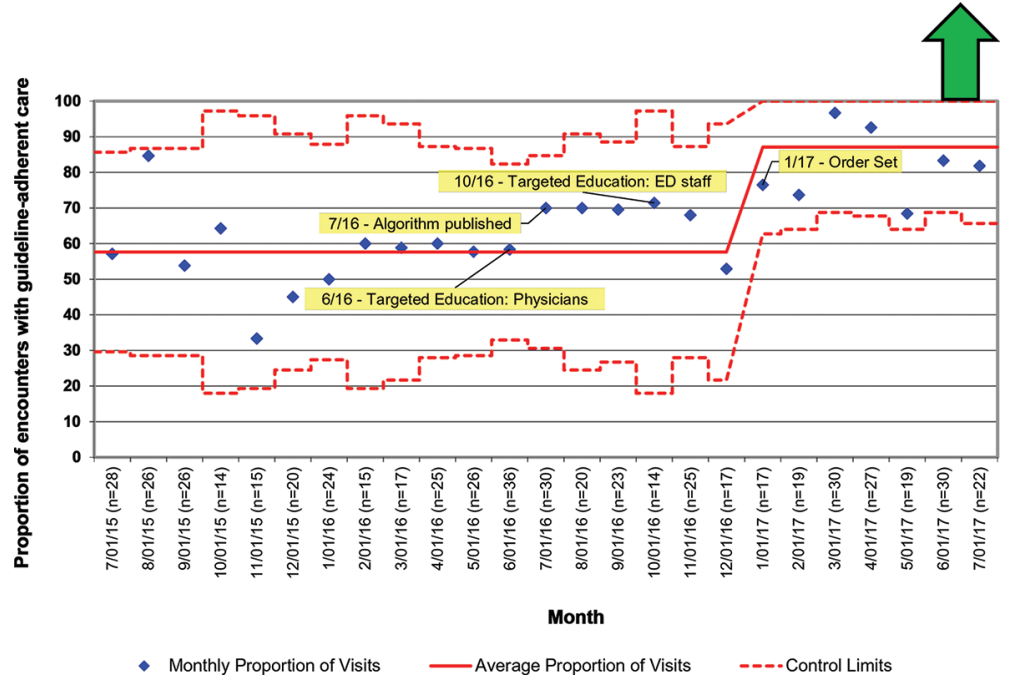

Abstract 985 Figure 2 Proportion of encounters for alleged sexual abuse with guideline-adherent care July 2015 - July 2017 


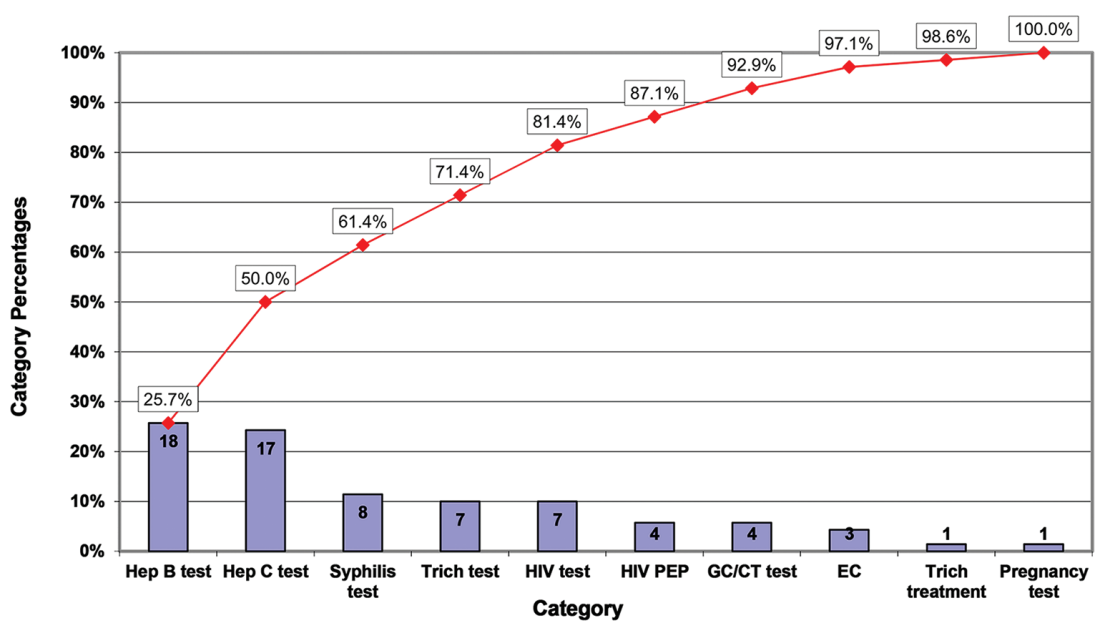

$\square$ Category Quantities \& Percentages

Abstract 985 Figure 3 Causes of non-adherence to guidline for evaluation and management of alleged sexual abuse by category Jan 2017 - June 2017, $\mathrm{n}=23$

encounters in which care adhered to guideline recommendations. Data were abstracted from the records of all patient encounters evaluated in the PED for reported sexual abuse.

Results We analysed 567 patient encounters for reported sexual abuse over 24 months. A statistical process control chart depicting the proportion of encounters with guideline-adherent care (Figure 2) illustrates special cause variation and a shift in the centerline from $57 \%$ to $87 \%$ which has been sustained for 7 months. We categorised reasons for non-adherence on a Pareto chart (Figure 3).

Conclusions Using improvement methodology, we successfully increased guideline-adherent evaluation and management of patients presenting for sexual abuse. Targeted education and an electronic order set were associated with improved adherence to a novel care guideline.

\section{REDUCING UNNECESSARY IV STARTS IN CHILDREN WITH DIABETES PRESENTING TO THE EMERGENCY DEPARTMENT}

Shannon Baumer-Mouradian, Matt Gray, Franklin Chang, Peter Wolfgram, David Brousseau, Catherine Ferguson. Medical College of Wisconsin, US

\subsection{6/bmjoq-2017-IHI.20}

Background Unnecessary medical interventions prolong emergency department (ED) stays and increase costs. We found that $83 \%$ of children with diabetes mellitus (DM) presenting to the ED not in diabetic ketoacidosis (DKA) underwent unnecessary IV placement.

Objectives We aimed to decrease IV placements to $20 \%$ within 18 months for children presenting to the ED with known DM not meeting DKA criteria.

Methods This QI project was conducted in a tertiary care paediatric ED and included children with known DM. Plando-study-act cycles included point-of-care (POC) testing, order panel use, and DKA clinical care and nursing guidelines.
Outcome measures, analysed on statistical process control charts, included number of IV starts and time to first bicarbonate result. The percent of patients receiving unnecessary IV starts was analysed using the Chi-square test. Process measures included rate of POC testing and order panel utilisation. Results Between January 2015 and July 2017, 294 DM patients were evaluated for DKA. 168 patients (57\%) did not meet DKA criteria. In those patients without DKA, the overall number of unnecessary IV starts decreased from $83 \%$ preinterventions to $41 \%$ post-interventions $(p<0.001$; Fig 1$)$. In the same 168 patients, mean time to first bicarbonate decreased from 78 to $29 \mathrm{~min}(62 \%)$ after implementation of all four interventions (Fig 2). Use of POC testing and order panels increased from zero to $92 \%$ and $75 \%$, respectively.

Conclusions Using QI methodology, we achieved a meaningful reduction in unnecessary IV starts and time to DKA determination in patients presenting with known DM found not to have DKA.

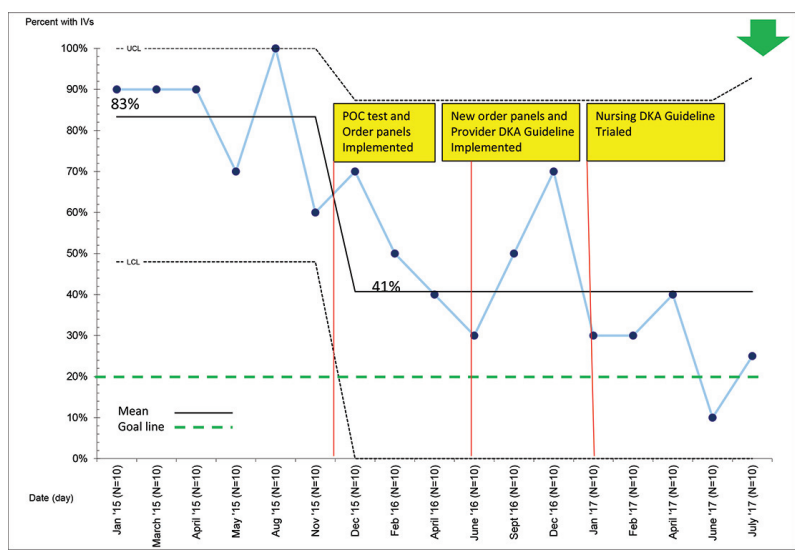

Abstract 998 Figure $1 \quad P$ Chart: proportion of patients with IV start (Pts. with DM without DKA) 